\title{
DEMOGRAPHICS OF BULGE TYPES WITHIN 11 Mpc AND IMPLICATIONS FOR GALAXY EVOLUTION
}

\author{
DAVID B. FISHER ${ }^{1,2}$ AND NIV DRORY ${ }^{3,4}$ \\ ${ }^{1}$ Laboratory of Millimeter Astronomy, University of Maryland, College Park, MD 29742, USA; dbfisher@astro.umd.edu \\ ${ }^{2}$ Department of Astronomy, The University of Texas at Austin, 1 University Station, C1400, Austin, TX 78712, USA \\ ${ }^{3}$ Instituto de Astronomía, Universidad Nacional Autónoma de México, A. P. 70-264, 04510 México, D. F., Mexico \\ ${ }^{4}$ Max-Planck-Institut für Extraterrestrische Physik, Giessenbachstraße, 85748 Garching, Germany \\ Received 2011 February 3; accepted 2011 March 29; published 2011 May 12
}

\begin{abstract}
We present an inventory of galaxy bulge types (elliptical galaxy, classical bulge, pseudobulge, and bulgeless galaxy) in a volume-limited sample within the local $11 \mathrm{Mpc}$ sphere using Spitzer $3.6 \mu \mathrm{m}$ and Hubble Space Telescope data. We find that whether counting by number, star formation rate, or stellar mass, the dominant galaxy type in the local universe has pure disk characteristics (either hosting a pseudobulge or being bulgeless). Galaxies that contain either a pseudobulge or no bulge combine to account for over $80 \%$ of the number of galaxies above a stellar mass of $10^{9} M_{\odot}$. Classical bulges and elliptical galaxies account for $\sim 1 / 4$, and disks for $\sim 3 / 4$ of the stellar mass in the local $11 \mathrm{Mpc}$. About 2/3 of all star formation in the local volume takes place in galaxies with pseudobulges. Looking at the fraction of galaxies with different bulge types as a function of stellar mass, we find that the frequency of classical bulges strongly increases with stellar mass, and comes to dominate above $10^{10.5} M_{\odot}$. Galaxies with pseudobulges dominate at $10^{9.5}-10^{10.5} M_{\odot}$. Yet lower-mass galaxies are most likely to be bulgeless. If pseudobulges are not a product of mergers, then the frequency of pseudobulges in the local universe poses a challenge for galaxy evolution models.
\end{abstract}

Key words: galaxies: evolution - galaxies: fundamental parameters - galaxies: general - galaxies: ISM - galaxies: photometry - galaxies: spiral

Online-only material: machine-readable table

\section{INTRODUCTION}

Hierarchical galaxy evolution models (e.g., White \& Rees 1978; Cole et al. 1994) rely on the assumption that bulgeto-total ratios increase directly, and exclusively, from merging (reviewed in Baugh 2006). This has been justified by the ability of simulations of mergers to reproduce properties of ellipticals (e.g., Cox et al. 2006; Naab et al. 2006), and the extrapolation motivated by observations of notable galaxies (e.g., M 31) that bulges are similar to ellipticals.

Yet, there is a dichotomy in the properties of bulges and possibly in their formation mechanisms. Some bulges are similar to elliptical galaxies (classical bulges), and other bulges resemble disks (pseudobulges; for reviews see Kormendy \& Kennicutt 2004; Combes 2009). Cursory analysis suggests that simulations producing bulge-disk galaxies (e.g., Governato et al. 2009) are likely not making pseudobulges.

Many authors propose that disk-like bulges form through internal secular evolution of the disk (for reviews see Kormendy \& Kennicutt 2004; Athanassoula 2005). Fisher \& Drory (2008, 2010) show that pseudobulges have Sérsic index $n<2$ and do not follow projections of the fundamental plane of elliptical galaxies, adding evidence that pseudobulges are physically different from classical bulges (which have $n>2$ ) and ellipticals. Fisher et al. (2009) find that pseudobulges commonly have high enough star formation rates (SFRs) to have built their stellar mass within the typical lifetime of a disk. Furthermore, correlations between bulge and disk properties such as stellar age (Peletier \& Balcells 1996) and radial size (Fisher \& Drory 2008) may result from a formative link between pseudobulges and their surrounding disk. Indeed, Fisher \& Drory (2010) find that the only property that correlates with the half-light radius of pseudobulges is the outer disk scale length.

Heller et al. (2007) show that significant gaseous inflow occurs across the central kpc during bar lifetimes. Bureau
\& Freeman (1999) show evidence that boxy/peanut-shaped bulges are the result of bar-buckling in disks. Boxy bulges are found in over $40 \%$ of edge-on galaxies (Lütticke et al. 2000), thus implying that a significant number of bulges may owe their origin to disk phenomena. We caution that secular evolution and accretion/merging are not mutually exclusive (Bournaud \& Combes 2002). Fisher \& Drory (2010) find that some pseudobulges ( $\lesssim 13 \%$ of their sample) could house a small classical bulge and still maintain a low Sérsic index.

How common are pseudobulges? Drory \& Fisher (2007) find that classical bulges are exclusively found in red-sequence galaxies, and imply that pseudobulges are at least as common as blue, Sa-Sc galaxies. Kormendy et al. (2010) find that in the local $8 \mathrm{Mpc}, 11$ of 19 galaxies with $V_{c}>150 \mathrm{~km} \mathrm{~s}^{-1}$ show no evidence for a classical bulge; however, this is a small sample that does not allow a study of the mass dependence of the frequency of pseudobulges. Weinzirl et al. (2009) show that traditional semi-analytic models of galaxy formation cannot account for the observed number of small bulges. This discrepancy may be a manifestation of the bulge dichotomy, since pseudobulges are more likely to be in low $B / T$ galaxies (Fisher et al. 2009). However, many pseudobulges have $B / T>0.2$ (Fisher \& Drory 2008, 2010).

In this Letter, we study the abundance of pseudobulges and classical bulges in the local universe. We determine bulge types on a sample including all non-edge-on galaxies having $B<15$ within $11 \mathrm{Mpc}\left(M_{B}<-15.2\right)$ and estimate the dependence of pseudobulge frequency on galaxy mass and SFR.

\section{METHODS}

We select a representative volume-limited sample of nonedge-on $\left(i<80^{\circ}\right)$ galaxies within $11 \mathrm{Mpc}$ from the Kennicutt et al. (2008) survey, complete for spirals to $B=15 \mathrm{mag}$ (corresponding to $M_{B}=-15.2$ ). We require Galactic latitude 
Table 1

Sample Data

\begin{tabular}{|c|c|c|c|c|c|c|c|c|c|c|}
\hline $\begin{array}{l}\text { Galaxy } \\
\text { Name }\end{array}$ & Category $^{\mathrm{a}}$ & $T$ & $\begin{array}{c}\text { Dist. } \\
(\mathrm{Mpc})\end{array}$ & $\begin{array}{c}M_{B} \\
(\mathrm{mag})\end{array}$ & $\begin{array}{c}\log \left(M_{*, \text { total }}\right) \\
\left(M_{\odot}\right)\end{array}$ & $\begin{array}{l}\log \left(\psi_{\text {total }}\right) \\
\left(M_{\odot} \mathrm{yr}^{-1}\right)\end{array}$ & $\begin{array}{c}\text { SFR } \\
\text { Method }\end{array}$ & $B / T$ & $\begin{array}{l}\text { Sérsic } \\
\text { Index }\end{array}$ & $\begin{array}{l}\log \left(\psi_{1 \mathrm{kpc}}\right) \\
\left(M_{\odot} \mathrm{yr}^{-1}\right)\end{array}$ \\
\hline NGC 6744 & $\mathrm{C}$ & 4 & 9.4 & -21.2 & 10.36 & -0.27 & $\mathrm{UV}, 24$ & 0.15 & $3.2 \pm 1.1$ & -2.33 \\
\hline NGC 0224 & $\mathrm{C}$ & 3 & 0.8 & -21.2 & 10.62 & -1.85 & 24 & 0.48 & $2.1 \pm 0.5$ & -2.04 \\
\hline NGC 4258 & $\mathrm{C}^{\mathrm{c}}$ & 4 & 8.0 & -21.0 & 10.49 & $\ldots$ & $\ldots$ & 0.11 & $2.8 \pm 0.6$ & $\ldots$ \\
\hline NGC 5055 & $\mathrm{P}$ & 4 & 7.5 & -20.5 & 10.48 & -0.10 & $\mathrm{UV}, 24$ & 0.19 & $1.3 \pm 1.4$ & -1.19 \\
\hline
\end{tabular}

Notes.

${ }^{\text {a }}$ E: elliptical galaxy; C: classical bulge; P: pseudobulge; nb/d: no bulge/dwarf; M: advanced stage merger.

${ }^{b}$ NGC 5194 and NGC 5195 are currently interacting.

${ }^{\mathrm{c}}$ Categorized as classical bulge due to Sérsic index despite nuclear morphology.

${ }^{\mathrm{d}}$ Morphology strongly indicates pseudobulge, despite high Sérsic index.

(This table is available in its entirety in a machine-readable form in the online journal. A portion is shown here for guidance regarding its form and content.)

$|b|>20^{\circ}$. We take $B_{T}$ values from de Vaucouleurs et al. (1991) and HyperLEDA ${ }^{5}$ in order of preference. Since the Kennicutt et al. (2008) sample does not cover early-type galaxies, we add these from Tonry et al. (2001), Tully \& Fisher (1988), and HyperLEDA using the same magnitude and Galactic latitude cuts. Because bulge diagnosis is not reliable on edge-on galaxies, we exclude disks with inclination greater than $80^{\circ}$. This selection may overemphasize the number of $E$ galaxies by $10 \%$ as they are not flattened. We adopt distances from Kennicutt et al. (2008) augmenting missing data from Tonry et al. (2001), Tully et al. (2009), and Tully \& Fisher (1988). Magnitudes and colors are corrected for extinction (Schlegel et al. 1998) and galaxy inclination in the usual manner. The final sample contains 320 galaxies. The full sample and measured quantities are listed in Table 1.

We decompose the major-axis near-IR surface brightness (SB) profile of 97 bright $\left(M_{B}<-16 \mathrm{mag}\right)$ and non $\mathrm{Sm} / \mathrm{Irr}$ galaxies at $3.6 \mu \mathrm{m}$ (2MASS $K$ band for six galaxies) into a Sérsic-function bulge and exponential outer disk. Nonexponential disk components (e.g., bars and rings) are masked. Most of our decompositions are taken from Fisher \& Drory (2010). This analysis has been used in many publications including Fisher \& Drory $(2008,2010)$ and Kormendy et al. (2009). The Sérsic index, $n$, is used to diagnose bulges into pseudo- $(n<2)$ and classical $(n>2)$ bulges (see Fisher \& Drory 2008 for a discussion). For those bulges with $n \sim 2$, we supplement bulge identification with nuclear morphology from Hubble Space Telescope (HST) images. Ellipticals are assigned $B / T=1$. Galaxies in which the decomposition yields $B / T<0.01$ are assigned $B / T=0$ and are called "bulgeless." We determine total luminosity by integrating the near-IR SB profile and convert to stellar mass using RC $3 B-V$ color as described in Fisher et al. (2009), following Bell \& de Jong (2001). Seven bright galaxies have no $B-V$ recorded and for these we substitute the average color of their Hubble type.

We assume that the 223 faint $\left(M_{B}>-16 \mathrm{mag}\right)$ or $\mathrm{Sm} / \mathrm{Irr}$ galaxies in our sample are bulgeless. Most have no usable nearIR data; we therefore use $M_{B}$ in conjunction with $B-V$ to determine stellar mass. One hundred and twenty-three do not have a measurement of $B-V$ and we again use the mean color of their Hubble type instead. For a handful of galaxies we test this against masses determined from near-IR flux, finding good agreement.

Available means of measuring SFR in our sample include GALEX FUV luminosity, $\mathrm{H} \alpha$ luminosity, and $24 \mu \mathrm{m}$ dust

\footnotetext{
5 http://leda.univ-lyon1.fr/
}

emission; linear combination of either $\mathrm{H} \alpha$ or UV (unobscured light) with $24 \mu \mathrm{m}$ (extincted light) is the most robust.

In galaxies fainter than $M_{B}=-16 \mathrm{mag}$, Lee et al. (2009) find that the UV SFR is systematically higher than that from other tracers, possibly due to differences in the stellar initial mass function. Therefore, we calculate the SFR from $\mathrm{H} \alpha$ and FUV according to Kennicutt (1998) and take the higher of the two values.

For 78 of the 97 bright galaxies, we measure the total SFR and the SFR within the central $1 \mathrm{kpc}$ by linearly combining the $24 \mu \mathrm{m}$ and GALEX FUV data (Leroy et al. 2008; Fisher et al. 2009), SFR $=a \times[L(\mathrm{FUV})+b \times L(24)]$, where $a$ and $b$ are constants calibrated against Kennicutt et al. (2009). The 19 remaining galaxies lack GALEX data. For six of these, we measure SFR by linearly combining $24 \mu \mathrm{m}$ with total $\mathrm{H} \alpha$ luminosity of Kennicutt et al. (2008), SFR $\propto L(\mathrm{H} \alpha)+a_{24} \times$ $L(24)$, according to Kennicutt et al. (2009). The SFR of the central $1 \mathrm{kpc}$ is measured with $24 \mu \mathrm{m}$ alone as SFR $\propto L(24)^{0.885}$ following Calzetti et al. (2007). Four galaxies have data at $24 \mu \mathrm{m}$ only; for these we follow Fisher et al. (2009). One galaxy has only $\mathrm{H} \alpha$ and one has only UV data; there we use the single band flux (SFR $\propto L(\mathrm{H} \alpha)$ or SFR $\propto L(\mathrm{FUV}))$ following Kennicutt (1998), and we cannot measure the luminosity of the central kpc. Finally, seven of the bright galaxies have no data available for measuring SFR. The method applied to calculate SFR for each galaxy is noted in Table 1 .

Uncertainties in stellar mass and SFR are dominated by the scatter in the calibration of measured fluxes to physical quantities. The calibration error for stellar mass is 0.12 dex for near-IR flux and $0.16 \mathrm{dex}$ for $M_{B}$. The calibration error for SFR is roughly $15 \%$ for data combining $\mathrm{H} \alpha+24 \mu \mathrm{m}$ and $\mathrm{FUV}+24 \mu \mathrm{m}$, and is closer to $20 \%$ for data using FUV or $\mathrm{H} \alpha$.

\section{RESULTS}

Before discussing our results, we call attention to the environmental bias inherent in studying galaxies in the local $11 \mathrm{Mpc}$ volume due to the low density of that region (reviewed in Peebles \& Nusser 2010). For comparison, Kormendy et al. (2009) find that $2 / 3$ of all stellar mass in the Virgo cluster is in elliptical galaxies alone.

Bulge number statistics. Galaxies with either a pseudobulge or no bulge are the most common among bright galaxies. Restricting ourselves to galaxies more massive than $10^{9} \mathrm{M}_{\odot}$, we find that only $17 \% \pm 10 \%$ are galaxies with an observed classical bulge (including elliptical galaxies), $45 \% \pm 12 \%$ are galaxies with pseudobulges, $35 \% \pm 12 \%$ are disk galaxies with 


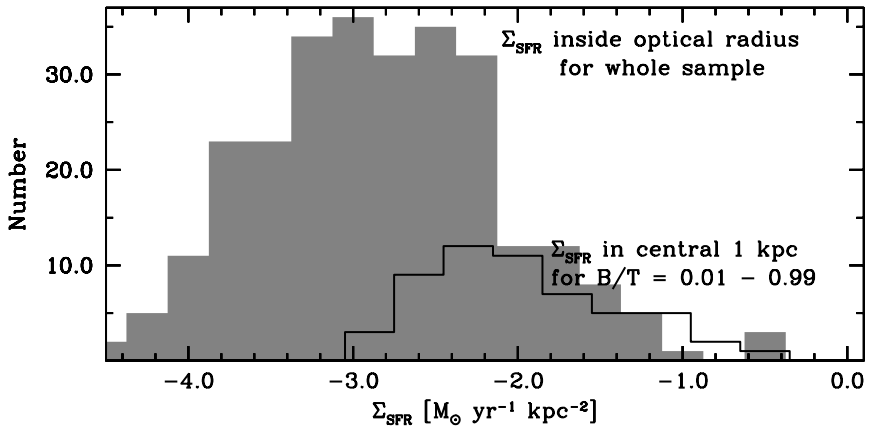

Figure 1. Distribution of SFR density $\Sigma_{\mathrm{SF}}(r<1 \mathrm{kpc})$ for bulges (black line). For comparison, we also show the SFR density of entire galaxies ( $\Sigma_{\mathrm{SF}}$ (total); gray shaded region).

$B / T<0.01$, and under $3 \%$ are galaxies currently undergoing major merging (NGC 4490, NGC 1487, NGC 2537). Quoted errors are Poisson uncertainties. Dwarf and irregular galaxies comprise $\sim 70 \%$ of all galaxies having stellar mass lower than $10^{9} M_{\odot}$ within $11 \mathrm{Mpc}$. However, they only account for $\sim 2 \%$ of the stellar mass in the same volume.

Star formation in bulge-disk galaxies. Sixty-one percent of the star formation (SF) in the local $11 \mathrm{Mpc}$ is in galaxies with pseudobulges. A non-negligible $13 \%$ of the total SF in our volume occurs in the central kpc of bulge-disk galaxies. Figure 1 shows the distribution of SFR surface densities $\left(\Sigma_{\mathrm{SF}}\right)$ of entire galaxies and the central kpc of bulge-disk galaxies. It is clear that high $\Sigma_{\mathrm{SF}}$ in the central kpc of bulge-disk galaxies is extremely common when compared to global SFR densities. In our sample, we find that $46 \% \pm 9 \%$ of galaxies with bulge-to-total ratios in the range $0.01 \leqslant B / T<1$ have $\Sigma_{\mathrm{SF}}>10^{-2} M_{\odot} \mathrm{yr}^{-1} \mathrm{kpc}^{-2}$; only $33 \% \pm 9 \%$ of entire galaxies have $\Sigma_{\mathrm{SF}}>10^{-2} M_{\odot} \mathrm{yr}^{-1} \mathrm{kpc}^{-2}$ inside the optical radius. In the bulge sample, 11 bulges do not have data to determine the SFR. If these have low SFR, the fraction of bulges with high $\Sigma_{\mathrm{SF}}$ decreases to $35 \% \pm 10 \%$.

Stellar masses. Figure 2 shows the stellar-mass distribution of galaxies with pseudobulges, classical bulges and ellipticals (combined), bulgeless galaxies, and the whole sample. Bulgeless galaxies tend to be lower in mass and dominate the distribution up to $M_{*} \sim 10^{9.5} M_{\odot}$. Pseudobulges dominate intermediate mass range from $M_{*} \gtrsim \times 10^{9.5}-10^{10.5} M_{\odot}$. Classical bulges tend to be in more massive galaxies. Galaxies with either a pseudobulge or no bulge combine to account for $56 \% \pm 12 \%$ of the stellar mass of galaxies within $11 \mathrm{Mpc}$. Finally, we calculate the total mass in classical bulges by using the $B / T$ from the bulge-disk decompositions. These values should be treated as estimates, since they assume the same $M / L$ for both the bulge and disk, hence likely underestimating the classical bulge mass. Classical bulges and E galaxies account for $\sim 1 / 4$ of the stellar mass in the local $11 \mathrm{Mpc}$, and disks account for $\sim 3 / 4$ of the stellar mass.

\section{DISCUSSION}

We show that galaxies with pseudobulges are the most common type of bright galaxy in the local $11 \mathrm{Mpc}$ volume. The set of galaxies including pseudobulge and bulgeless galaxies accounts for just over $1 / 2$ of the mass in stars in the local volume. Roughly $2 / 3$ of new stars are made in galaxies with pseudobulges. Whether counting by number, mass, or by present-day SF, the dominant mode of galaxy evolution in the present-day local universe is that which occurs in galaxies without classical bulges. These results are therefore

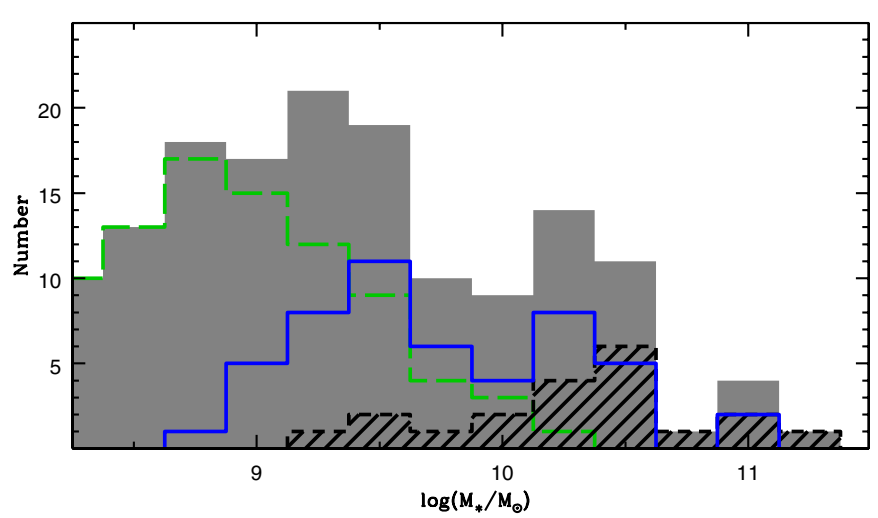

Figure 2. Distribution of galaxy stellar mass in galaxies with pseudobulges (blue line), elliptical galaxies and galaxies with classical bulges (short-dashed line), bulgeless galaxies (green long-dashed line), and the full sample (gray shaded region). Note the full range of stellar masses in our sample is not shown.

in agreement with the observed correlation of bulge type with galaxy properties such as color (Drory \& Fisher 2007).

We find that classical bulges and elliptical galaxies combined account for $\sim 1 / 4$ of the stellar mass within $11 \mathrm{Mpc}$. Therefore, $3 / 4$ of the stellar mass in the local $11 \mathrm{Mpc}$ is in disks (combining all mass in pseudobulges, disks around classical bulges and pseudobulges, and bulgeless galaxies). Recall that in cluster environments, $2 / 3$ of the stellar mass is in elliptical galaxies alone (Kormendy et al. 2009). Thus, the process driving the distribution of bulge types appears to be a strong function of environment.

We show that in the majority of bulge-disk galaxies, the central kpc has high SFR surface density (compared with the SFR density for entire galaxies). If a merger drives enhanced SFR for 1 Gyr (Cox et al. 2008), and if fewer than $10 \%$ of giant galaxies experience merging each Gyr (e.g., Jogee et al. 2009), then episodic SF cannot account for the frequency of enhanced SF observed in our sample, and thus the SF in the centers of (pseudo)bulges is likely not episodic or merger driven. The frequency of enhanced SF in bulges is thus further evidence that bulges are generating new stars through long-term, non-episodic processes.

Finally, in Figure 3, we estimate the relative frequency of classical bulges (including elliptical galaxies), pseudobulges, all bulges, and galaxies with no bulge within $11 \mathrm{Mpc}$ as a function of galaxy stellar mass. To account for the possibility of composite systems, we estimate an upper bound for the frequency of classical bulges: we include all those bulges that satisfy the criteria to be called classical and elliptical galaxies, add all galaxies presently in strong interactions (NGC 4490, NGC 1487, NGC 2537, and NGC 5194A and B), and we estimate the possible number of galaxies with composite (pseudo+classical) bulges. Fisher \& Drory (2010) find that models of bulges in which the total bulge light is composed of a high and low Sérsic index component are not inconsistent with decompositions of real bright low-specific-SFR pseudobulges. Consistent with these results, we select all pseudobulges with stellar mass $M_{\text {pseudo }}>10^{9} M_{\odot}$ and specific SFR $<0.03 \mathrm{Gyr}^{-1}$ as candidate composite bulges. For the interacting galaxies, we make the assumption that a merger will result in an elliptical galaxy and thus $B / T=1$.

Figure 3 shows that the frequency of pseudobulges and classical bulges in the local universe is strongly dependent on galaxy mass. Pure disk galaxies and those galaxies with pseudobulges are the most common type of galaxy for stellar 


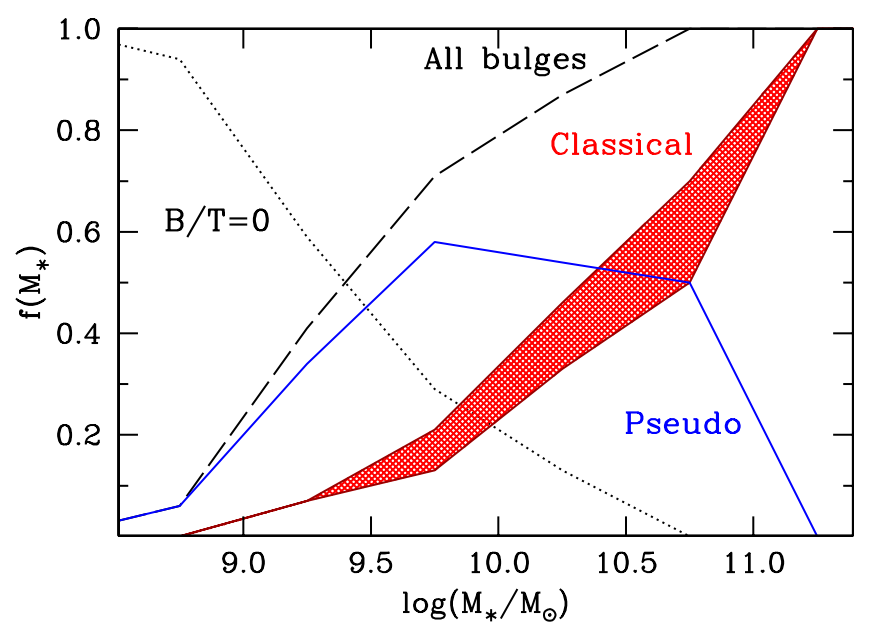

Figure 3. Relative number of galaxies with classical bulges and elliptical galaxies (red lines), galaxies containing pseudobulges (blue line), all disk-bulge galaxies (black dashed line), and bulgeless galaxies (black dotted line) as a function of galaxy stellar mass.

mass $M_{*} \leqslant 10^{10} M_{\odot}$. Elliptical galaxies and galaxies with classical bulges are the majority of galaxies with $M_{*} \geqslant 10^{10.5} M_{\odot}$. However, since galaxies with $M_{*} \geqslant 10^{10.5} M_{\odot}$ only make up $4 \%$ of bright galaxies in the local volume, galaxies with pseudobulges and those with no bulge remain the dominant type of bright galaxy by number. Dynamical evidence suggests that the Milky Way (not included in the sample) does not contain a classical bulge (Shen et al. 2010); its stellar mass places it right at the transition, $M_{*, \mathrm{MW}} \sim$ $10^{10.5} M_{\odot}$. Therefore, the massive galaxies in the Local Group comprise a pseudobulge galaxy (Milky Way), a classical bulge galaxy (M 31), and a bulgeless disk galaxy (M 33).

The simulation of the evolution of galaxies in a $\Lambda \mathrm{CDM}$ universe by Croft et al. (2009) provides a good model for comparison. As is normally the case, in this simulation $B / T$ is only increased through the merging process. Croft et al. find that in massive galaxies $\left(\gtrsim 10^{9} M_{\odot}\right)$ located in low-density environments (i.e., field galaxies), $40 \%-50 \%$ are bulge dominated $(B / T>80 \%)$. In the local $11 \mathrm{Mpc}$ only $23 \% \pm 5 \%$ of galaxies contain classical bulges at any bulge-to-total ratio (including ellipticals and ongoing mergers), and only $5 \%$ have $B / T>80 \%$. If we assume that the simulation in Croft et al. (2009) only produces classical bulges, then the number of classical bulges in the local universe is much smaller than in a typical galaxy evolution simulation.

Recently, Hopkins et al. (2009b) showed that if $B / T$ is a function of both merger mass ratio and gas fraction in the progenitive merger, then the distribution of $B / T$ for all galaxies is recovered. However, this agreement relies entirely on the interpretation of pseudobulges as merger products (contrary to observational evidence). In our sample, the fraction of classicalbulge light in galaxies less massive than $M_{*} \sim 10^{10} M_{\odot}$ is very low, $B / T \lesssim 5 \%$. If pseudobulges are not merger products, but rather disk components, then models continue to produce too much mass in bulges.

We conclude that pseudobulges and internal bulge growth through SF are present in the majority of giant disk galaxies in the local $11 \mathrm{Mpc}$ volume. If we make the assumption that pseudobulges are not direct merger products, then the number of pseudobulges poses a challenge for models of galaxy evolution. Given that very old stellar populations are commonly observed in spiral galaxies (MacArthur et al. 2009), holding off disk galaxy formation until lower redshifts does not appear to be the solution. The problem is that, as we understand them now, mergers in recent epochs are likely to increase $B / T$ and heat the disk, thereby reducing the secular inward flow of gas in disks, and possibly destroying a pre-existing pseudobulge in a disk galaxy. Therefore, either the merging process does not disrupt disks as easily as simple calculations suggest (see Hopkins et al. 2009a; Moster et al. 2010), or there are fewer galaxy mergers in recent epochs in the universe than simulations suggest.

D.B.F. acknowledges support from University of Maryland, as well as $\mathbf{J}$ Kormendy and the University of Texas at Austin. N.D. and D.B.F. thank the Max-Planck Society for support during this project. We also thank Shardha Jogee, Karl Gebhardt, Neal Evans, John Kormendy, and Ralf Bender for their helpful comments and support during the writing of this work. This work is based on observations made with the Spitzer Space Telescope, which is operated by the Jet Propulsion Laboratory, California Institute of Technology under a contract with NASA. Support for this work was provided by NASA through an award issued by JPL/Caltech. D.B.F. acknowledges support by the National Science Foundation under grant AST 06-07490. Some of the data presented in this Letter were obtained from the Multi-mission Archive at the Space Telescope Science Institute (MAST). STScI is operated by the Association of Universities for Research in Astronomy, Inc., under NASA contract NAS526555. Support for MAST for non-HST data is provided by the NASA Office of Space Science via grant NAG5-7584 and by other grants and contracts.

\section{REFERENCES}

Athanassoula, E. 2005, MNRAS, 358, 1477

Baugh, C. M. 2006, Rep. Prog. Phys., 69, 3101

Bell, E. F., \& de Jong, R. S. 2001, ApJ, 550, 212

Bournaud, F., \& Combes, F. 2002, A\&A, 392, 83

Bureau, M., \& Freeman, K. C. 1999, AJ, 118, 126

Calzetti, D., et al. 2007, ApJ, 666, 870

Cole, S., Aragon-Salamanca, A., Frenk, C. S., Navarro, J. F., \& Zepf, S. E. 1994, MNRAS, 271, 781

Combes, F. 2010, in ASP Conf. Ser. 419, Galaxy Evolution: Emerging Insights and Future Challenges, ed. S. Jogee et al. (San Francisco, CA: ASP), 31

Cox, T. J., Jonsson, P., Primack, J. R., \& Somerville, R. S. 2006, MNRAS, 373, 1013

Cox, T. J., Jonsson, P., Somerville, R. S., Primack, J. R., \& Dekel, A. 2008, MNRAS, 384, 386

Croft, R. A. C., Di Matteo, T., Springel, V., \& Hernquist, L. 2009, MNRAS, 400,43

de Vaucouleurs, G., de Vaucouleurs, A., Corwin, H. G., Jr., Buta, R. J., Paturel, G., \& Fouque, P. 1991, Third Reference Catalogue of Bright Galaxies (Vol. 1-3, XII; Berlin: Springer)

Drory, N., \& Fisher, D. B. 2007, ApJ, 664, 640

Fisher, D. B., \& Drory, N. 2008, AJ, 136, 773

Fisher, D. B., \& Drory, N. 2010, ApJ, 716, 942

Fisher, D. B., Drory, N., \& Fabricius, M. H. 2009, ApJ, 697, 630

Governato, F., et al. 2009, MNRAS, 398, 312

Heller, C. H., Shlosman, I., \& Athanassoula, E. 2007, ApJ, 671, 226

Hopkins, P. F., Cox, T. J., Younger, J. D., \& Hernquist, L. 2009a, ApJ, 691, 1168

Hopkins, P. F., et al. 2009b, MNRAS, 397, 802

Jogee, S., et al. 2009, ApJ, 697, 1971

Kennicutt, R. C., et al. 2009, ApJ, 703, 1672

Kennicutt, R. C., Jr. 1998, ARA\&A, 36, 189

Kennicutt, R. C., Jr., Lee, J. C., Funes, S. J. J. G., Sakai, S., \& Akiyama, S. 2008, ApJS, 178, 247

Kormendy, J., Drory, N., Bender, R., \& Cornell, M. E. 2010, ApJ, 723, 54

Kormendy, J., Fisher, D. B., Cornell, M. E., \& Bender, R. 2009, ApJS, 182, 216

Kormendy, J., \& Kennicutt, R. C. 2004, ARA\&A, 42, 603 
Lee, J. C., et al. 2009, ApJ, 706, 599

Leroy, A. K., Walter, F., Brinks, E., Bigiel, F., de Blok, W. J. G., Madore, B., \& Thornley, M. D. 2008, AJ, 136, 2782

Lütticke, R., Dettmar, R., \& Pohlen, M. 2000, A\&AS, 145, 405

MacArthur, L. A., González, J. J., \& Courteau, S. 2009, MNRAS, 395, 28

Moster, B. P., Macciò, A. V., Somerville, R. S., Johansson, P. H., \& Naab, T. 2010, MNRAS, 403, 1009

Naab, T., Khochfar, S., \& Burkert, A. 2006, ApJ, 636, L81

Peebles, P. J. E., \& Nusser, A. 2010, Nature, 465, 565

Peletier, R. F., \& Balcells, M. 1996, AJ, 111, 2238

Schlegel, D. J., Finkbeiner, D. P., \& Davis, M. 1998, ApJ, 500, 525
Shen, J., Rich, R. M., Kormendy, J., Howard, C. D., De Propris, R., \& Kunder, A. 2010, ApJ, 720, L72

Tonry, J. L., Dressler, A., Blakeslee, J. P., Ajhar, E. A., Fletcher, A. B., Luppino, G. A., Metzger, M. R., \& Moore, C. B. 2001, ApJ, 546, 681

Tully, R. B., \& Fisher, J. R. 1988, Catalog of Nearby Galaxies (Cambridge: Cambridge Univ. Press)

Tully, R. B., Rizzi, L., Shaya, E. J., Courtois, H. M., Makarov, D. I., \& Jacobs, B. A. 2009, AJ, 138, 323

Weinzirl, T., Jogee, S., Khochfar, S., Burkert, A., \& Kormendy, J. 2009, ApJ, 696, 411

White, S. D. M., \& Rees, M. J. 1978, MNRAS, 183, 341 